\title{
A Quantitative Analysis of the Spatiotemporal Pattern of Transient Receptor Potential Gene Expression in the Developing Mouse Cochlea
}

\author{
Yukako Asai, ${ }^{1} \mathrm{Jeffrey}^{\mathrm{R} .}$ Holt, ${ }^{1}$ and Gwenaëlle S. G. GÉléoc ${ }^{1}$ \\ ${ }^{1}$ Departments of Neuroscience and Otolaryngology, University of Virginia School of Medicine, MR4, Room 5122, Box 801392, \\ Charlottesville, VA 22908-1392, USA
}

Received: 28 July 2009; Accepted: 25 September 2009; Online publication: 16 October 2009

\begin{abstract}
TRP genes encode a diverse family of ion channels which have been implicated in many sensory functions. Because several TRP channels have similar properties to the elusive hair cell transduction channel, recent attention has focused on TRP gene expression in the inner ear. At least four TRP genes are known to be expressed in hair cells: TRPC3, TRPV4, TRPA1, and TRPML3. However, there is little evidence supporting any of these as a component of the transduction complex. Other less well-characterized TRP channels are expressed in the inner ear, in particular, within the organ of Corti. Because of their potential role in sensory function, we investigated the developmental expression of RNA that encodes all 33 TRP subunits as well as several splice variants. We designed a quantitative PCR screen using cochlear samples acquired before, during, and after the time when mechanotransduction is acquired in sensory hair cells (embryonic day 17 to postnatal day 8). Cochleas, which included the organ of Corti, stria vascularis, and Reissner's membrane, were subdivided into four equal quadrants which allowed for regional comparison during development. Expression of RNA transcripts that encoded 33 TRP subunits plus several splice forms and beta-actin were quantified in 28 samples for a total of 1,092 individual measurements, each done in triplicate. We detected RNA that encoded all TRP channels except two: TRPC7 and TRPM8. The largest changes in RNA expression were for TRPA1 (>100-fold), TRPP3 (>50-fold), and TRPC5.2 (>20-fold)
\end{abstract}

\footnotetext{
Electronic supplementary material The online version of this article (doi:10.1007/s10162-009-0193-8) contains supplementary material, which is available to authorized users.

Correspondence to: Gwenaëlle S. G. Géléoc · Departments of Neuroscience and Otolaryngology - University of Virginia School of Medicine · MR4, Room 5122, Box 801392, Charlottesville, VA 22908-1392, USA. Telephone: +1-434-2439942; fax: +1-434-9824380; email: gg3h@virginia.edu
}

which suggested that these subunits may contribute to normal cochlear function. Furthermore, the screen revealed TRPP3 and PKD1L3 RNA expression patterns that were correlated with the acquisition of sensory transduction in outer hair cells (Lelli et al., J Neurophysiol. 101:2961-2973, 2009). Numerous spatiotemporal expression gradients were identified many of which may contribute to the normal functional development of the mouse cochlea.

Keywords: mouse, inner ear, organ of corti, TRP channels, development, quantitative PCR

\section{INTRODUCTION}

The Transient Receptor Potential (TRP) superfamily encodes over 33 distinct proteins which have been implicated in numerous cellular and sensory functions including detection of chemical, thermal, and mechanical stimuli (Montell 2005; Damann et al. 2008). The mammalian family is divided into six subfamilies: TRPC (Canonical), TRPM (Melastatin), TRPV (Vanilloid), TRPA (Ankyrin), TRPP (Polycystin), and TRPML (Mucolipin). Over the past decade, interest in TRP expression in the ear has risen in part due to the ongoing search for the elusive hair cell transduction channel which has biophysical properties consistent with those of several TRP channels. The transduction channel is a nonselective cation channel with a conductance of about $100 \mathrm{pS}$, permeable to calcium and sensitive to inhibition by TRP channel antagonists, ruthenium red, and lanthanum in turtle (Farris et al. 2004) and mouse (Géléoc and Holt, unpublished). Several TRP channel subunits have been presented as hair cell transduction channel candidates, including TRPN1 (Walker et al. 2000), TRPV4 (Liedtke et al. 2000), TRPA1 (Corey et al. 2004), and recently, TRPML3 (Grimm et al. 2007; Nagata et al. 2008; 
van Aken et al. 2008). Yet, targeted gene deletion of single TRP genes has not revealed deficits in hair cell mechanotransduction. However, since TRP channels may consist of heteromultimers, the hair cell transduction channel may be composed of more than one type of TRP subunit; hence, upregulation of subunits that remain may compensate for absent subunits. Furthermore, it is possible that the identity of the hair cell transduction channel remains hidden within the pool of TRP genes not yet examined. Alternatively, the hair cell transduction channel may be composed of ion channels that do not belong to the TRP family.

Despite the lack of evidence supporting a role for TRP channels in hair cell transduction, it is clear that TRP gene expression is critical for normal auditory function. Analysis of two TRP genes, TRPV4 and TRPML3, expressed in the ear revealed important nonsensory roles for TRPs in hearing and balance. TRPV4 is expressed in hair cells, stria vascularis, and vestibular dark cells (Liedtke et al. 2000; Takumida et al. 2005), and disruption of TRPV4 causes delayed-onset hearing loss and increases susceptibility to acoustic injury (Tabuchi et al. 2005). TRPML3 is expressed in sensory hair cells and in the marginal cells of the stria vascularis (Di Palma et al. 2002; Nagata et al. 2008; Van Aken et al. 2008). Mutations in TRPML3 lead to deafness in varitintwaddler (Va) mice due to defects in stria vascularis and hair cells. The Va mutation induces constitutive activation of inward currents which causes cellular degeneration (Nagata et al. 2008; Van Aken et al. 2008).

Other TRP channels, such as TRPC3, are known to be expressed in the ear and may play important roles as well. Conventional RT-PCR analysis of TRP genes in the mouse organ of Corti revealed expression of 19 TRPs (Cuajungco et al. 2007). A separate large-scale analysis of ion channel expression identified 21 TRP genes expressed in the inner ears of species that ranged from zebrafish to humans (Gabashvili et al. 2007) (See Table 1). However, neither screen provided a quantitative description of TRP expression. As such, we opted to examine TRP RNA expression in the mouse cochlea during the first postnatal week, when hair cells become functional (Lelli et al. 2009). We performed a quantitative analysis of RNAs that code for 33 TRP subunits and their alternative splice forms in four cochlear quadrants between embryonic day 17 (E17) and postnatal day 8 (P8). We present the results of our analysis and discuss several TRP genes with spatiotemporal expression patterns that may be worthy of further investigation.

\section{MATERIAL AND METHODS}

\section{Tissue harvest}

Inner ear organs from embryonic (E) and early postnatal (P) Swiss Webster mice (Taconic Farms,
Germantown, NY) were harvested and used to generate cDNA banks as described previously (Lelli et al. 2009). Briefly, a total of six to 20 ears were dissected at each developmental stage: E17, E18, P0, P2, P4, P6, and $\mathrm{P} 8$. For each cochlea dissected, the entire length of the organ was measured using an Axiocam MRC5 video camera and Zeiss Image Browser (Zeiss, Oberkochen, Germany). Subsequently, the organ was divided into four equal quadrants: basal end (BA), mid-basal (MB), mid-apical (MA), and apical end (AP). Sections from the same quarter and developmental stage were combined to prepare total RNA. Each section included the organ of Corti, the stria vascularis, and the Reissner's membrane. All protocols used for this study have been approved by the Animal Care Committee of the University of Virginia (Protocol \#3123).

\section{cDNA bank preparation and quantitative PCR}

cDNA banks for quantitative PCR (qPCR) were prepared as described previously (Lelli et al. 2009). Total RNA of each quarter was purified using RNAqueous Micro kit (Ambion Inc., Austin, TX) followed by a treatment with Turbo DNA-free (Ambion Inc., Austin, TX). Samples were analyzed using a bioanalyzer (Agilent Technologies, Santa Clara, CA) to determine RNA integrity. Only samples with RNA integrity number (RIN) greater than 8.0 and free of contamination with genomic DNA were used for further procedures (Supplemental Fig. 1). A threshold RIN of eight was chosen to minimize the likelihood of RNA degradation (Fleige and Pfaffl 2006). One microgram of total RNA was used for linear amplification using RiboAmp RNA amplification kit (Arcturus, Mountain View, CA). The amplified RNA from each quarter was reverse-transcribed for the cDNA bank with an iScript kit (Biorad, Hercules, $\mathrm{CA})$. Expression of TRP genes, as well as the housekeeping gene $\beta$ actin $(A c t b)$ was analyzed by single color real-time PCR with SYBR GreenER qPCR reagent (Invitrogen, Carlsbad, CA) and iCycler (Biorad, Hercules, CA). Primer sets were designed within the region of $1 \mathrm{~kb}$ upstream from the polyA site using the PrimerQuest Software from Integrated DNA Technologies (http://www.idtdna.com) and validated on control tissue (see Supplemental Table 1). When splice variants existed that did not share this region, extra primer sets were designed (see Supplemental Table 1). Quantitative PCR reactions were performed according to the manufacturer's instruction (SYBR GreenER qPCR reagent, Invitrogen, Carlsbad, CA). Each reaction was executed in triplicate simultaneously and included primers at $200 \mathrm{nM}$ and cDNA generated from $12.5 \mathrm{ng}$ of amplified RNA. The cycle threshold (Ct) values of triplicates for each sample 


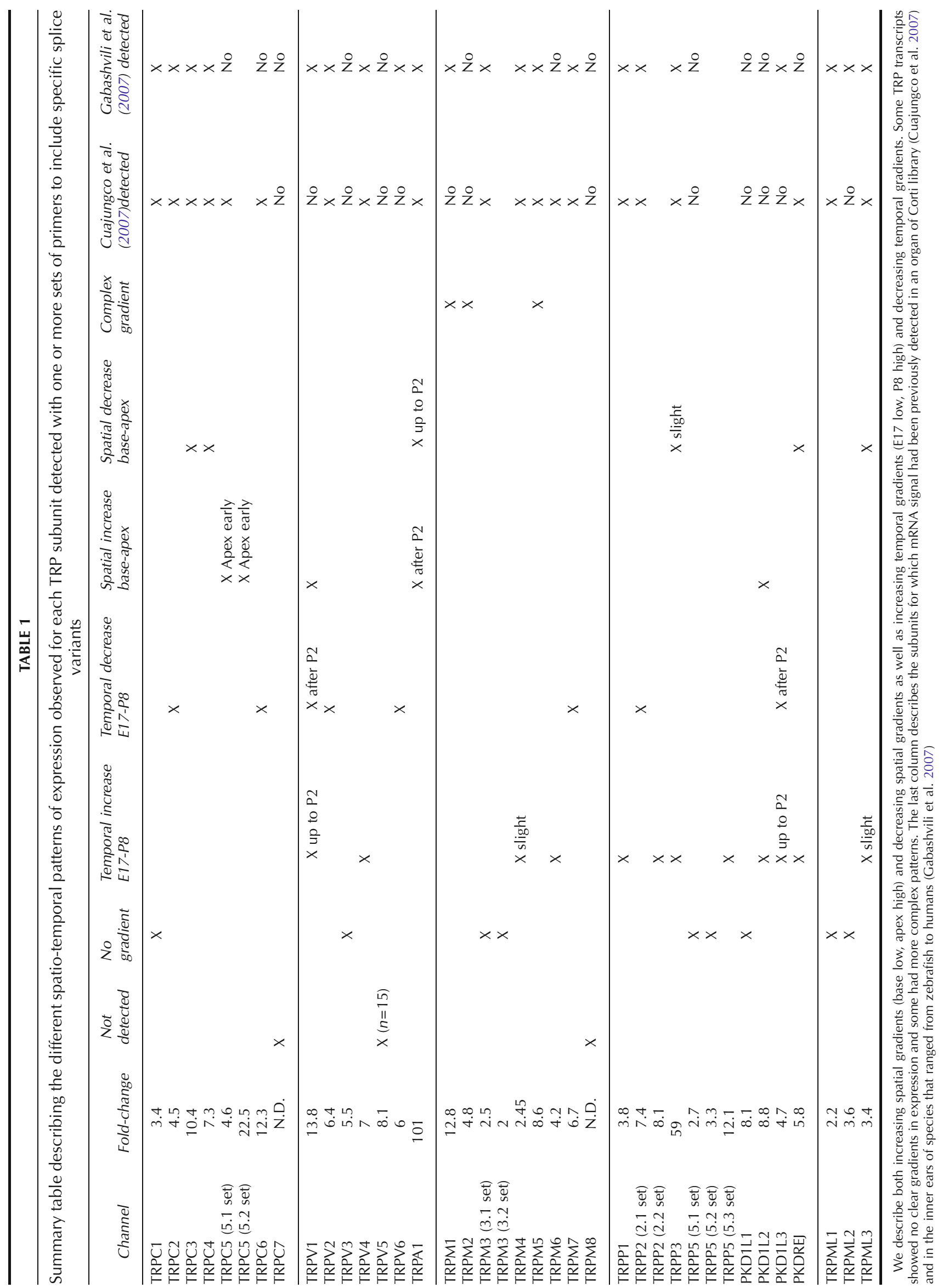


were averaged and normalized relative to the averaged $\mathrm{Ct}$ value of $\beta$ actin obtained from the same sample. The fold difference for each TRP gene expression level was calculated with the comparative $\Delta \mathrm{Ct}$ method and normalized to most immature quarter E17 apex as described previously (Lelli et al. 2009).

\section{RESULTS}

RNA samples were prepared from inner ear tissue harvested from four quadrants of the developing mouse cochlea between E17 and P8. We used quantitative RT-PCR to examine the relative RNA expression of 33 TRP genes and several alternative splice variants using a total of 38 primer sets. We did not determine the efficiency of each TRP primer set; therefore, direct comparison of expression level between different TRP subunits was not possible. Rather, relative RNA expression levels were calculated which allowed for comparison of expression levels for each of the 28 samples for a given TRP primer set. To calculate relative RNA expression, we normalized to beta-actin RNA expression level for each of the 28 samples. Each dataset was then normalized to the sample taken from the apex at E17, which, we reasoned, was the most immature. The results are presented in five consecutive figures in which TRP channels of the same or similar families are grouped together. The mean relative abundance values of for all datasets \pm standard deviation are included in Supplemental Table 2. In the following sections, we present the data for each family and note both spatial and temporal gradients of RNA expression for a number of TRP transcripts. We describe both increasing spatial gradients (base low, apex high) and decreasing spatial gradients as well as increasing temporal gradients (E17 low, P8 high) and decreasing temporal gradients. Some TRP transcripts showed no clear gradients in expression and some had more complex patterns.

\section{TRPC family}

There are seven canonical transient receptor potential channels (TRPC) subunits which form tetrameric, calcium-permeable ion channels, some of which can be activated by G-protein coupled receptors, the second messenger diacylglycerol DAG, and changes in intracellular calcium concentrations (Ramsey et al. 2006; Kiselyov and Patterson 2009). We analyzed expression of RNA coding for the seven TRPC subunits using specific primer probes including two for TRPC5 (-C5.1 and -C5.2) to detect alternative TRPC5 splice variants (Supplemental Table 1):
TRPC1, TRPC2, TRPC3, TRPC4, TRPC5.1, TRPC5.2, TRPC6, and TRPC7 (Fig. 1). All primer sets were validated on cerebellum extracts or $\mathrm{P} 4$ cochleas (Supplemental Table 1). No signal was detected for TRPC7, suggesting that if TRPC7 transcripts were present their expression levels were below our detection threshold. To better appreciate the range of expression levels during development and along the cochlea, we calculated the largest fold-change in expression observed for each TRP transcript regardless of quadrant or developmental stage. The largest spatiotemporal fold-change in expression for each TRPC channel were, respectively: -C1, 3.4; -C2, 4.5; -C3, 10.4; -C4, 7.3; -C5.1, 4.6; -C5.2, 22.5; and -C6, 12.3. TRPC1 expression was fairly stable amongst the 28 samples indicating no major change in expression. TRPC2 demonstrated a temporal decrease in expression across the organ. There was both a spatial and temporal decrease in expression of TRPC3 and TRPC4 such that both were more highly expressed in the base and at earlier stages. RNA coding for TRPC5 was higher in the apex at earlier stages (E17P2 for TRC5.1 and E17-E18 for TRPC5.2) but otherwise stable across the organ and during development. TRPC6 expression also decreased during development but remained relatively stable along the length of the cochlea.

\section{TRPM family}

The TRPM subfamily comprises eight mammalian members which play a role in various sensory and nonsensory functions. Except for TRPM4 and TRPM5, TRPM channels form $\mathrm{Ca}^{2+}$-permeable cation channels (for review, see Owsianik et al. 2006). TRPM3 splice variants possess different selectivity; some with high permeability to $\mathrm{Ca}^{2+}$ and $\mathrm{Mg}^{2+}$ and blocked by extracellular monovalent cations (Oberwinkler et al. 2005). As many as nine TRPM3 splice variants have been identified (Oberwinkler et al. 2005 and the Mouse Genome Database, Jackson Labs). We designed two primer sets (-M3.1 and -M3.2) which together allowed us to detect all TRPM3 splice variants. TRPM3.1 detected all splice variants but one and TRPM3.2 detected the remaining variant but may have also detected others (See Supplemental Table 1 for accession numbers). None of the TRPM3 primers detected non-TRPM3 transcripts. We, therefore, analyzed expression of RNA coding for eight TRPM subunits which included two primer sets designed to detect TRPM3 splice variants: TRPM1, TRPM2, TRPM3.1, TRPM3.2, TRPM4, TRPM5, TRPM6, TRPM7, TRPM8 (Fig. 2). Signals for all TRPM channels were detected in our preparation with the exception of TRPM8 which appeared not to be expressed in the cochlea. The largest spatiotemporal changes in expression for each TRPM channel were, respectively, -M1, 

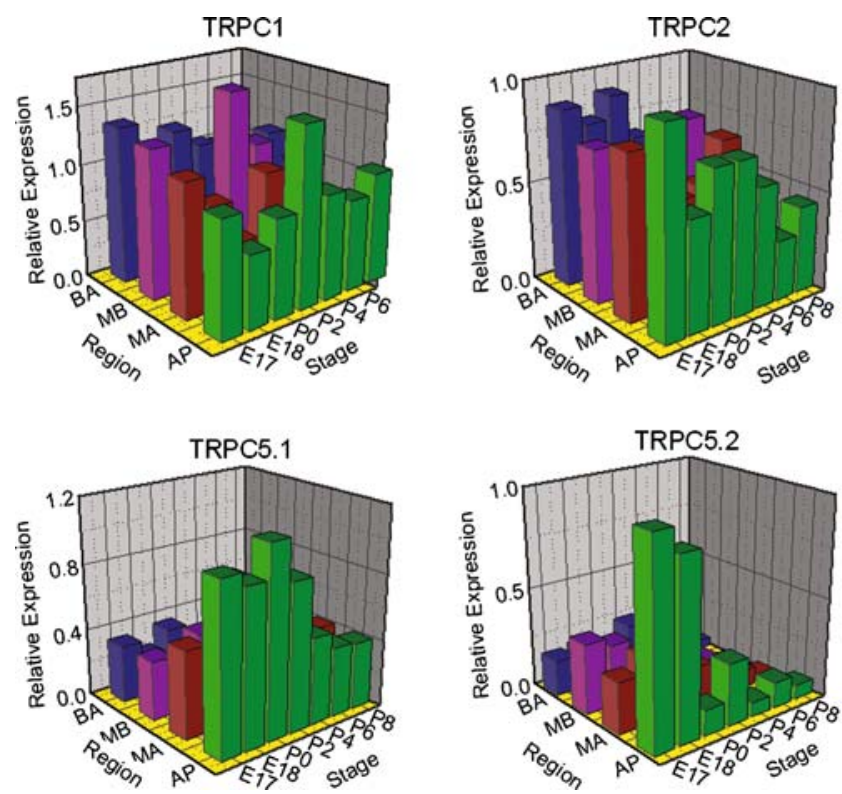

FIG. 1. RNA expression of TRPCs along the cochlea from E17 to P8. The 3D plot illustrates relative qPCR measurements of six TRPC subunits (TRPC1-TRPC6) normalized to beta-actin and the most immature stage E17 Apex (E17AP). Two sets of primers were used to

12.8; -M2, 4.8; -M3.1, 2.5; -M3.2, 2.0; -M4, 2.45; -M5, 8.6; -M6, 4.2; and -M7, 6.7. RNA coding for TRPM3 transcripts did not shown large changes in expression either across the organ or during development. TRPM4 showed little change in expression spatially but did display a slight increase in expression developmentally. Larger changes were observed for the other members of the family, in particular TRPM1 which was much more
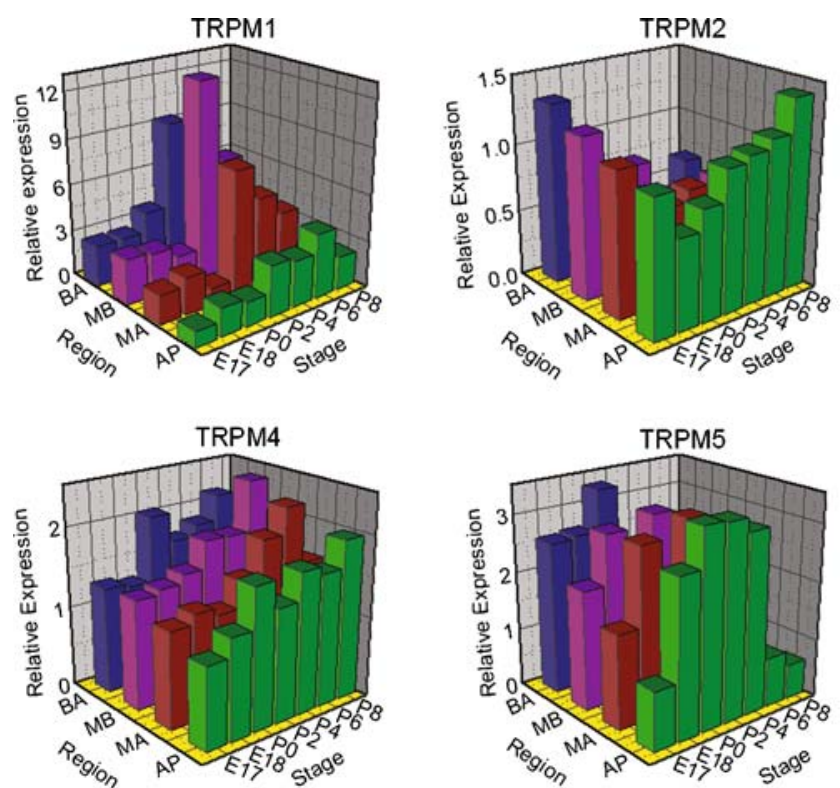

FIG. 2. RNA expression of TRPMs along the cochlea from E17 to P8; The 3D plot illustrates relative qPCR measurements of seven TRPM subunits (TRPM1-TRPM7) normalized to beta-actin and the most immature stage, E17AP. Two sets of primers were used to detect
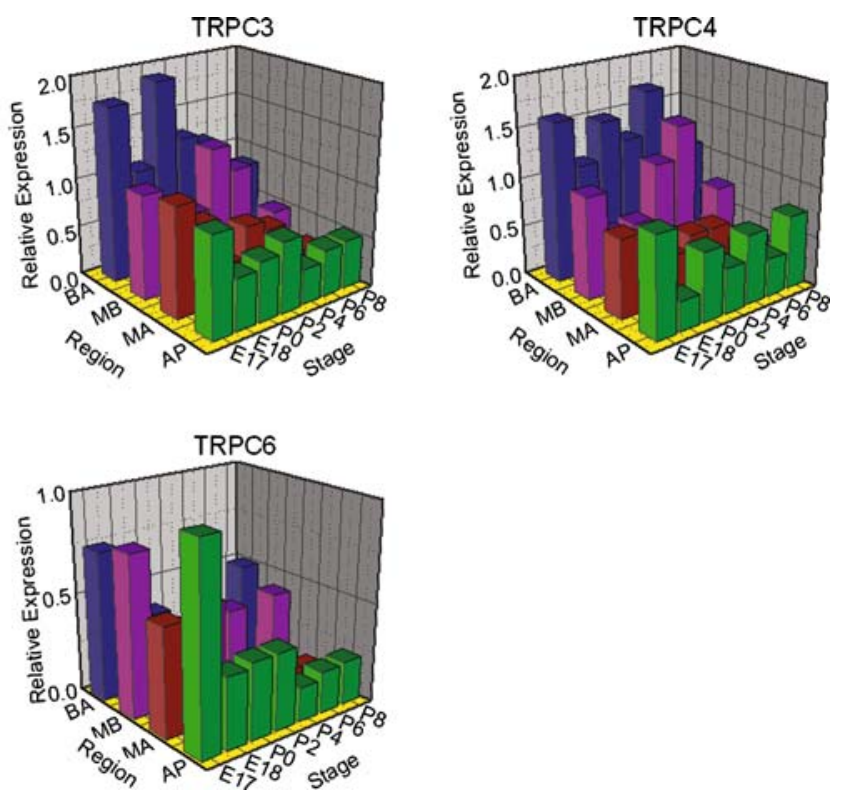

detect TRPC5 expressions, which are listed as TRPC5.1 and TRPC5.2. Corresponding numerical values \pm SD are listed in Supplemental Table 2. No signal was detected using a TRPC7specific primer set.

highly expressed at P2, particularly in the basal half. TRPM5 also showed significant spatiotemporal changes: a decreasing spatial gradient was evident at E17; expression then leveled off by E18-P0, after which it decreased dramatically first in the base at P2 then after $\mathrm{P} 4$ in the apex. The rise in TRPM5 expression coincided with the pattern of acquisition of transduction in the cochlea (Lelli et al. 2009). RNA coding for TRPM6 was
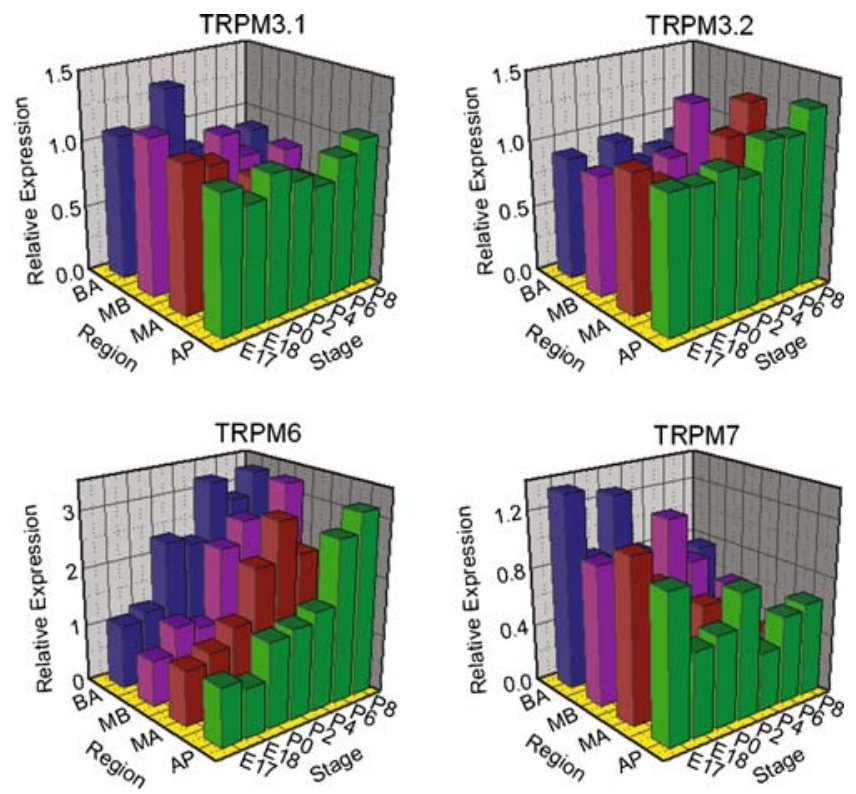

TRPM3 expressions which are listed as TRPM3.1 and TRPM3.2. Corresponding numerical values \pm SD are listed in Supplemental Table 2. No signal was detected using a TRPM8-specific primer set. 
relatively constant across the organ; however, an increase in expression was clear over the first postnatal week. TRPM7 expression decreased at older stages without any apparent spatial gradient. TRPM2 had a more complex expression pattern with a slight decreasing spatial gradient at E17; an increasing temporal gradient in the apex and a decreasing temporal gradient in the base.

\section{TRPV family}

Expression of six TRPVs, vanilloid receptor-like channels was analyzed. Members of the TRPV channel family have been shown to play a role in sensory transduction as well as in other non-sensory pathways. TRPV4 in particular has been shown to be expressed in inner and outer hair cells, vestibular hair cells, stria vascularis, and vestibular dark cells (Liedtke et al. 2000; Takumida et al. 2005). Our RNA expression screen revealed spatial and/or temporal gradients in expression for most of the TRPV channels (Fig. 3). TRPV5, however, was not detected for 15 of the 28 samples with none detected at $\mathrm{P} 4$ or P6. The largest spatiotemporal changes in expression for each TRPV channel were: -V1, 13.8; -V2, 6.4; -V3, 5.5; -V4, 7.0; -V5, 8.34; and -V6, 6.0. TRPV1 was more highly expressed in the basal quarter between P0 and P6. Smaller changes in expression were observed in the apex. TRPV2 mainly showed a developmental gradient with a decrease in expression during maturation of the organ. TRPV3 showed a peak in expression in the base at P0 but otherwise was relatively stable. TRPV4 presented a developmental gradient with a gradual increase from E17 to P0 in the base and E17 to P6 in the apex. TRPV6 displayed a decreasing gradient in expression with higher expression at E17 followed by a gradual decrease up to P8 all along the cochlea.

\section{TRPA1 channels}

Expression of the ankyrin repeat rich TRPA1 channel was also analyzed (Fig. 3). TRPA1 is of particular interest since it was presented as a candidate for the mechanotransduction channel in inner ear hair cells and in the lateral line (Corey et al. 2004). Subsequent work determined that TRPA1 is required for chemosensation in zebrafish and mammals, as well as cold and mechanical reception in mammals, but is not required for mechanosensory hair cell function in either mammals (Bautista et al. 2006; Kwan et al. 2006) or zebrafish (Prober et al. 2008). Interestingly, TRPA1 RNA expression remained fairly constant at the base but was elevated in the apex particularly around P0 and P2, around the time of acquisition of mechanotransduction in apical outer hair cells (Lelli et al. 2009). Of all the TRP channels examined in our screen, the largest foldchange in expression observed was for TRPA1 with a change of 101-fold between the lowest (P4BA) and highest RNA expression level (P2AP). Whether this steep spatial gradient in TRPA1 RNA expression is of functional significance for the developing cochlea remains to be determined (see "Discussion").
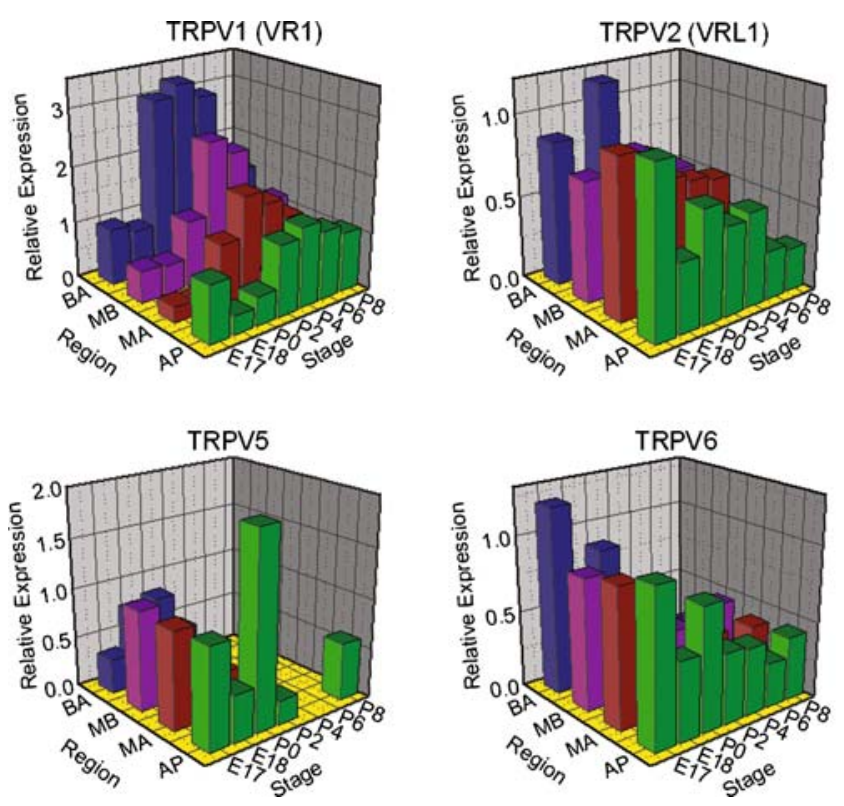

FIG. 3. RNA expression of TRPVs and TRPA1 along the cochlea from E17 to P8; The 3D plot illustrates relative qPCR measurements of six TRPV subunits (TRPV1-TRPV6) and TRPA1 normalized to beta-
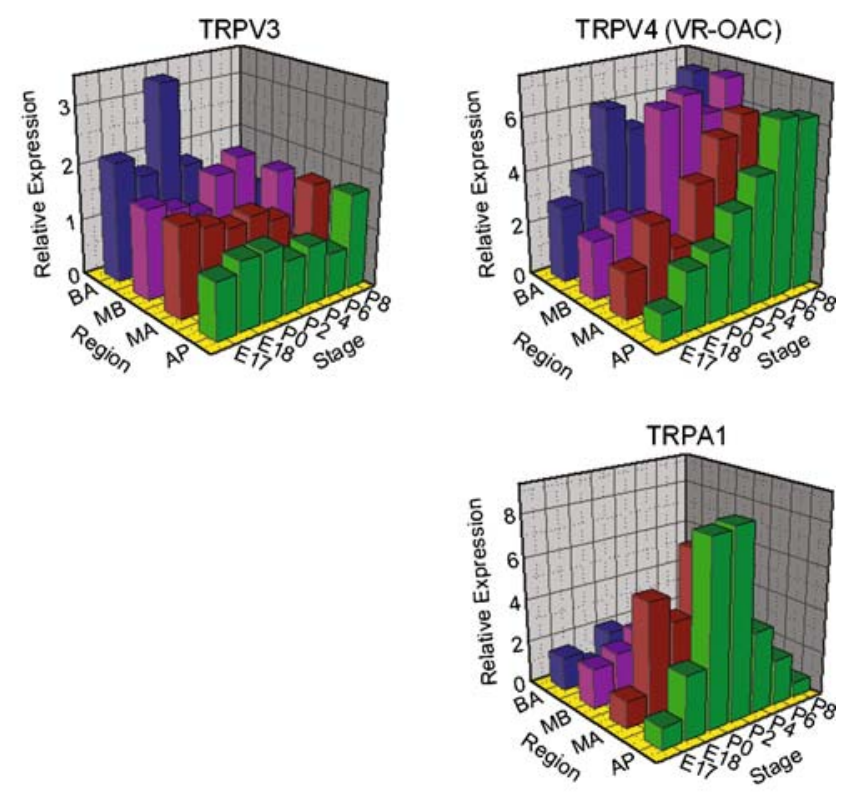

actin and the most immature stage, E17AP. Corresponding numerical values \pm SD are listed in Supplemental Table 2. 


\section{TRPP family}

The TRPP channels are polycystins originally named polycystic kidney disease (PKD) channels because mutations in these channels result in renal cyst formation. The TRPP family is large and divided in two subgroups: (1) the PKD1 like group which includes TRPP1, PKDREJ, PKD1L1, PKD1L2, and PKD1L3 and (2) the PKD2 like which includes pore forming proteins TRPP2, TRPP3 (PKD2L1), and TRPP5 (PKD2L2). TRPP1 subgroup members encode large accessory proteins. Figure 4 shows RNA expression for all the TRPP members. RNA expression was detected using specific probes (Supplemental Table 1) which included two different primer sets designed to detect variants of TRPP2 (-P2.1 and -P2.2) and three sets designed to detect variants of TRPP5 (-P5.1, -P5.2, -P5.3). The largest spatiotemporal changes in expression for each TRPP subunits were: -P1, 3.8; -P2.1, 7.4; -P2.2, 8.1; -P3, 59; -P5.1, 2.7; -P5.2, 3.3; -P5.3, 12.1; PKD1L1, 8.1; PKD1L2, 8.8; PKD1L3, 4.7; and PKDREJ, 5.8. TRPP3 RNA had the largest fold-change in
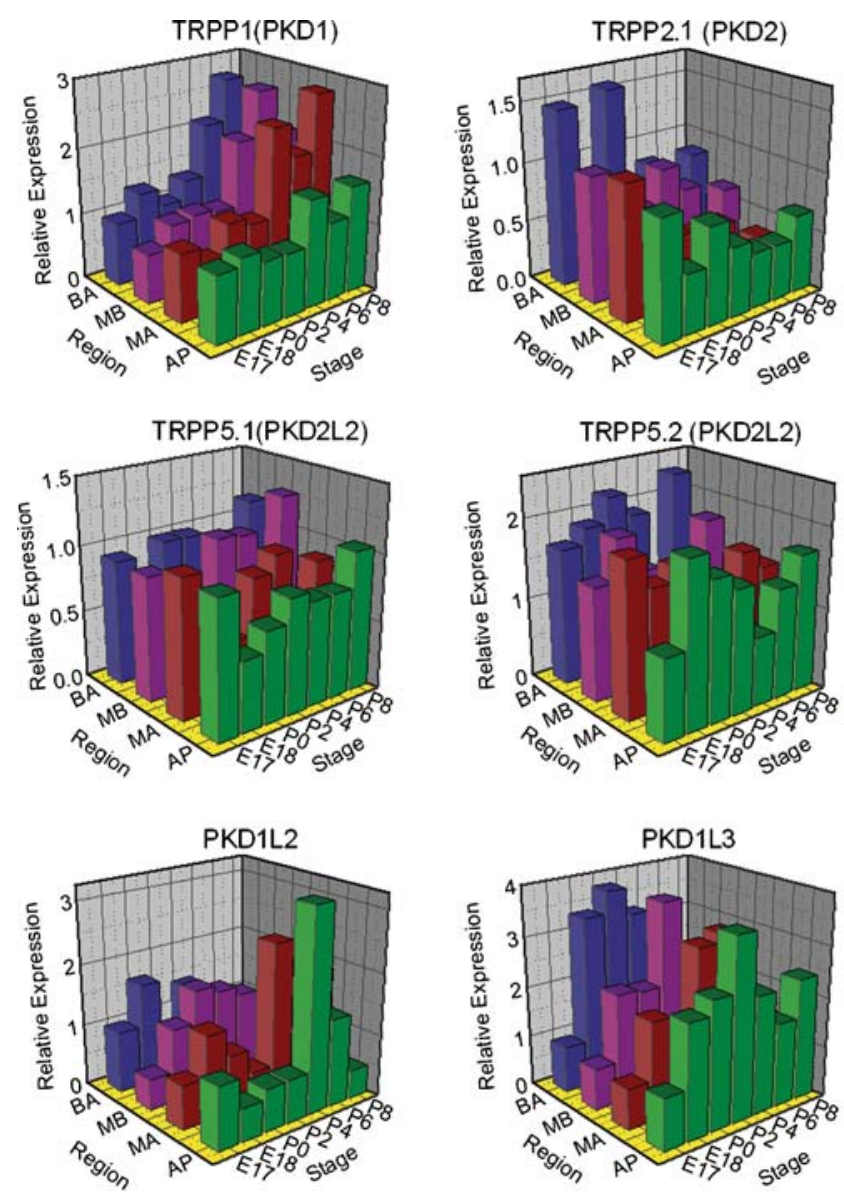

FIG. 4. RNA expression of TRPPs along the cochlea from E17 to P8; The 3D plot illustrates relative qPCR measurements of 8 TRPPs subunits (TRPP1-3, TRPP5, PKD1L1, PKD1L2, PKD1L3, and PKDREJ) normalized to beta-actin and E17AP. Two sets of primers were used to detect expression TRPP2 splice forms which are listed as TRPP2.1 expression for the TRPP family. The highest TRPP3 expression level was observed in the mid apical region at P2 and expression also increased in the basal half before P2. The pattern of TRPP3 RNA expression paralleled the gradient of acquisition of transduction in hair cells (Lelli et al. 2009). A similar pattern was observed for PKD1L3 although the change in RNA expression was less pronounced. TRPP1 and TRPP2.2 RNA signal did not show a substantial spatial gradient but did display an increasing temporal gradient, while RNA detected with the TRPP2.1 probe presented a slightly decreasing temporal gradient. TRPP5.2 and TRPP5.3 RNA signals were somewhat erratic and not easily characterized whereas TRPP5.1 RNA expression appeared relatively stable throughout the cochlea and the first postnatal week. PKD1L1 displayed a complex expression pattern, increasing in the apex and decreasing in the base during the first postnatal week. PKD1L2 also had a complex pattern with a prominent peak at P4 in the apex. Lastly, there was an increasing temporal gradient for PKDREJ expression which was more prominent in the base.
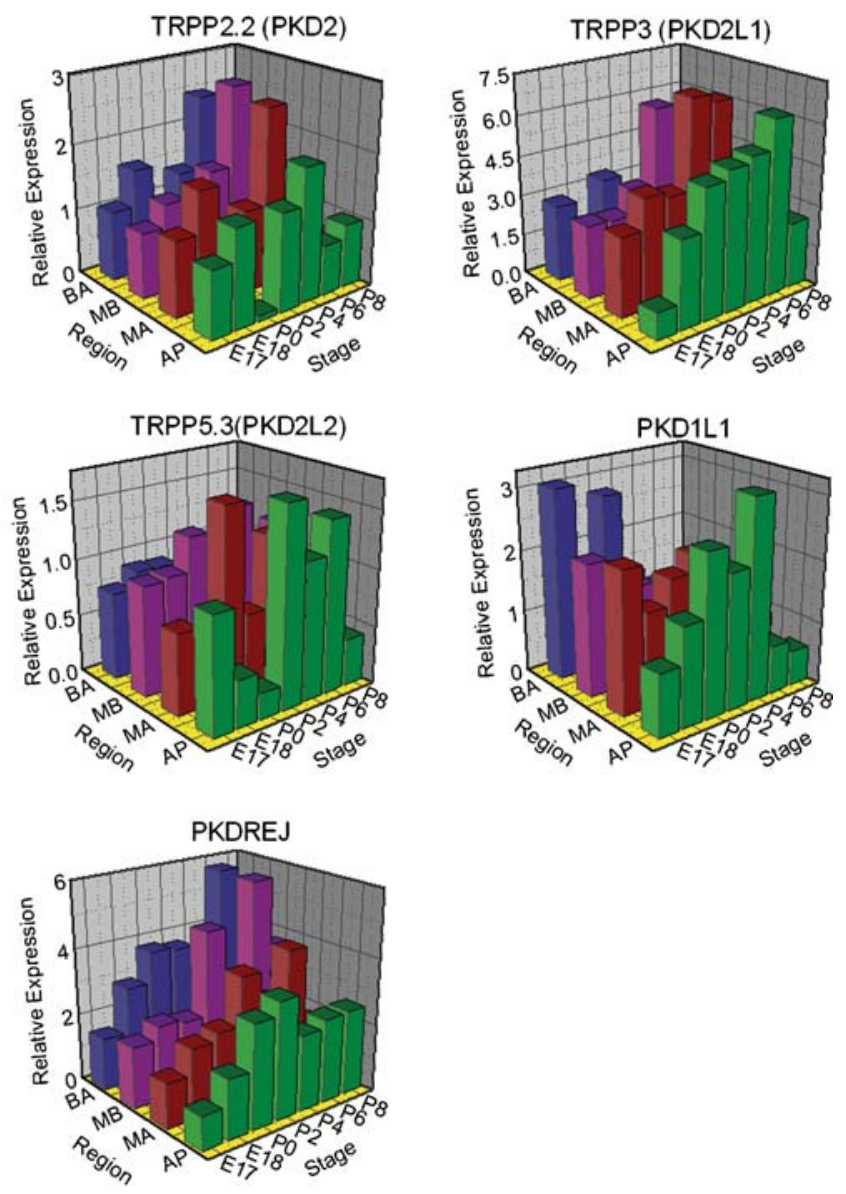

and TRPP2.2 (see Supplemental Table 1). Three sets of primers were used to detect TRPP5 splice forms which are listed as TRPP5.1, TRPP5.2, and TRPP5.3. Corresponding numerical values \pm SD are listed in Supplemental Table 2. 


\section{TRPML family}

TRPML family includes three members TRPML 1-3 of which one, TRPML3, is known to be expressed in the inner ear (Nagata et al. 2008; Van Aken et al. 2008; Grimm et al. 2009; Takumida and Anniko 2009). While we were able to detect signal that encoded all TRPML members (Fig. 5), the largest fold-change in expression remained low at 2.2 for -ML1, 3.6 for -ML2 and 3.4 for -ML3 between E17 and P8 along the cochlea. Although neither TRPML1 nor TRPML2 showed any clear expression gradients, TRPML3 displayed a slight spatiotemporal gradient.

\section{DISCUSSION}

The pattern of RNA expression for all 33 TRP subunits and several splice variants was analyzed using qPCR and RNA harvested from four cochlear quadrants at seven developmental stages between E17 and P8. The goal of the analysis was to identify spatiotemporal expression patterns for each TRP gene during maturation of the cochlea over the first postnatal week, when hair cells become functional, just prior to the onset of hearing. To optimize transcript integrity, RNA was harvested within the first few minutes of inner ear dissections. The organ of Corti, the tectorial membrane, the stria vascularis, and the Reissner's membrane were included in the samples. Integrity of all 28 RNA samples was confirmed and determined to be of high quality. In a previous study using the same 28 RNA samples we demonstrated that the expression pattern of RNA that encoded prestin (Lelli et al. 2009) matched the functional acquisition of somatic outer hair cell motility in developing sensory hair cells (Belyantseva et al. 2000; Abe et al. 2007), which raised confidence that our samples could yield functionally relevant RNA expression patterns. The advantage of a qPCR screen of this sort is that it is a very sensitive assay and allows for direct and quantitative comparison between samples. Although the screen did not permit expression level comparisons between differ- ent TRP subunits, it did allow us to compare expression levels between samples for a given TRP subunit and identify correlations between the spatiotemporal patterns of TRP expression and prior measures of cochlear function. As such, we present these data as an initial screen of TRP RNA spatiotemporal expression in the cochlea and intend that they may form a basis for future study of TRPs of particular interest. We not only highlight several TRP genes with noteworthy expression patterns but also note that the expression data may reveal other patterns or correlations worthy of further attention. Of course, it is important to bear in mind that in some cases, there may be little correlation between the spatiotemporal pattern of RNA expression and the functional expression of the protein. While a lack of correlation cannot be taken as evidence that a gene is not involved in a given cochlear function, a positive correlation can call attention to a particular TRP subunit. Furthermore, since RNA expression must precede the functional expression of any protein, RNA expression that turns on after functional acquisition of a given property can be used to rule out that RNA from further consideration as having a role in the function.

TRP subunits with no or little expression and/or no distinct expression pattern

The result of our screen demonstrated that RNAs encoding all but two TRP subunits, TRPC7 and TRPM8, were detected. TRPV5 expression was also low such that none was detected in half the samples. RNA transcripts for TRPC7, TRPM8, and TRPV5 were also undetected in a conventional RT-PCR screen of mouse organ of Corti (Cuajungco et al. 2007). Our analysis revealed nine other TRP channels that did not show substantial changes during the first postnatal week of development. The following nine TRPs either revealed no prominent spatiotemporal expression patterns or revealed little change in expression level during the time frame we examined: TRPC1, TRPV3, TRPM3.1, TRPM3.2, TRPP5.1, TRPP5.2, PKD1L1, TRPML1, and TRPML2. While we cannot take the
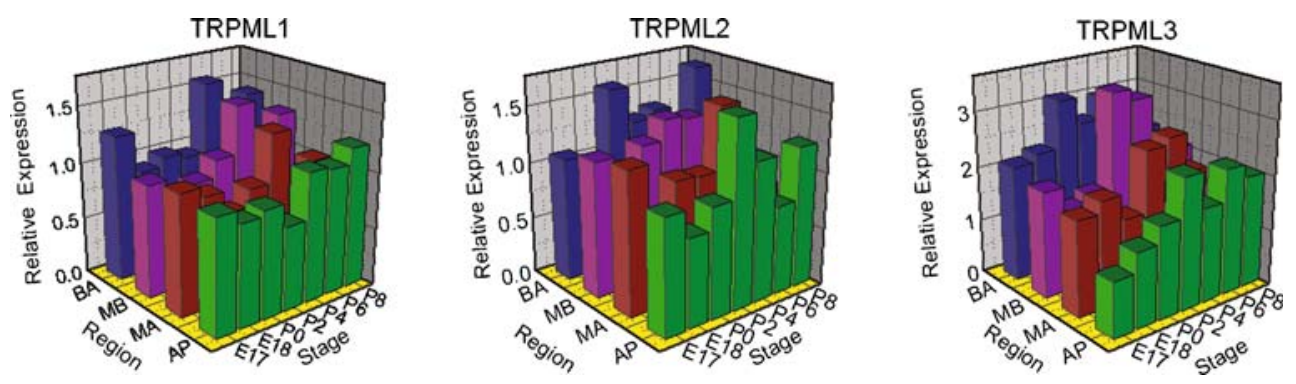

FIG. 5. RNA expression of TRPMLs along the cochlea from E17 to P8; the 3D plot illustrates relative qPCR measurements of 3 TRPML subunits (TRPML1-TRPML3) normalized to beta-actin and the most

immature stage E17 Apex (E17AP). Corresponding numerical values \pm SD are listed in Supplemental Table 2. 
data to mean that these 12 TRP subunits are inconsequential for normal cochlear function, the lack of correlation with functionally relevant aspects of cochlear development gives us no reason prioritize them for further investigation.

\section{TRP subunits previously shown to be expressed} in the inner ear

We were interested to examine the expression pattern of TRP channels previously identified and known to be expressed in the inner ear. TRPC3 has been shown to be expressed in cochlear hair cells and may contribute to calcium homeostasis (Raybould et al. 2007). Interestingly, Raybould et al. demonstrated a three-fold difference in TRPC3 currents between basal and apical outer cells of the guinea pig cochlea. We noted a similar three-fold basal to apical gradient in TRPC3 RNA, particularly around P0, further supporting the notion that our RNA expression data may correspond to functionally relevant measures of cochlear physiology.

Another notable TRP subunit, TRPA1, gained considerable attention when it was presented as a candidate for the mechanosensitive transduction channel (Corey et al. 2004). However, it was later ruled out as a candidate based on the lack of an auditory or vestibular deficit in TRPA1-deficient mice (Bautista et al. 2006; Kwan et al. 2006). To our surprise, TRPA1 RNA expression spiked over 100-fold in the apex at $\mathrm{P} 0$ and $\mathrm{P} 2$ relative to the base at $\mathrm{P} 4$. We confirmed the TRPA1 expression profile in preamplified RNA preparations. The range of expression of TRPA1 was well above the maximal change in expression of all other TRP subunits, TRPP3 being the nearest competitor with a 59-fold-change in expression. Although TRPA1-deficient mice do not shown any vestibular or hearing deficit, our data suggest that TRPA1 may play a significant role, particularly in the low frequency, apical end of the cochlea. However, since no deficits in hearing or hair cell transduction were detected even in the low frequency range, it remains possible that loss of TRPA1 expression in the knockout mice may have been compensated by upregulation of a functionally redundant protein. While TRP channels are known to form heteromultimers, it is not clear which TRP subunits can heteromultimerize with other TRP partners. If TRPA1 co-assembles with another TRP subunit, it is conceivable that the other subunit can compensate for the loss of TRPA1 in the mutant animals. Interestingly, analysis of our TRP expression data from wild-type mice revealed that TRPV1 followed an inverse pattern of expression, particularly around P0 to P2 with higher expression in the base. We find the inverse basal/apical gradient for TRPA1 and TRPV1 intriguing and wonder whether the two subunits might interact with varying stoichiometries and thereby confer a gradual change in functional properties along the length of the cochlea. While TRPA1 and TRPV1 co-assembly may seem unlikely, these two subunits are co-expressed in various cell types. Like TRPA1, TRPV1 subunits are cation channels expressed in nociceptive sensory neurons where they have been implicated in inflammatory pain (Caterina et al. 1997; Woolf and Costigan 1999; Montell 2005; Wang and Woolf 2005). Recent reports demonstrated that the TRPA1 and TRPV1 channels can interact and regulate the expression of the other (Akopian et al. 2007; Salas et al. 2009). Thus, it is conceivable that altered expression of one channel could affect expression of the other (Salas et al. 2009) and perhaps even compensate for loss of the other. If this relationship holds true in the cochlea, we hypothesize that there may be an upregulation of TRPV1 expression that could functionally compensate for the loss of TRPA1 expression in TRPA1deficient mouse cochleas.

TRPV4 has also been shown to be expressed in vertebrate cochlear hair cells and in stria vascularis (Liedtke et al. 2000). Our RNA expression screen revealed a spatiodevelopmental pattern such that an increase in expression occurred in the basal quarter from E17 to P0, up to P2 in the mid-basal quarter, and $\mathrm{P} 4-\mathrm{P} 6$ in the apical half. The basal-apical gradient that was observed during development follows the maturation of the organ. Because the TRPV4-deficient mice suffer from delayed-onset hearing loss, it is possible that TRPV4 channels play an important role in the mature organ, in which case we predict that TRPV4 expression may continue to rise after P8. TRPV4, TRPC1, and TRPC6 are frequently coexpressed in DRG neurons (Alessandri-Haber et al. 2009). In the cochlea, TRPC1 and TRPC6 did not have similar expression patterns. TRPC6, in particular, showed an opposing expression gradient suggesting that these channels might not be co-expressed within the same cells in the inner ear. Since our qPCR screen does not provide cellular resolution immunolocalization or in situ hybridization will be required to identify where within the cochlea TRP subunits are expressed and whether they are colocalized in the same cell types.

Although TRPML3 is expressed in the inner ear (Nagata et al. 2008; Van Aken et al. 2008; Grimm et al. 2009; Takumida and Anniko 2009), our expression screen did not reveal large changes in expression between E17 and P8. TRPML3 showed a shallow RNA expression gradient during development and across the organ. In situ hybridization data showed that TRPML3 RNA was widely detected in numerous cells of the cochlea (Nagata et al. 2008). Thus, the trend in RNA 
expression we observed cannot be attributed to any particular cell type as it represents the total change in expression across the entire cochlea which may obscure changes in subpopulations of cells within the cochlea.

\section{Other TRP subunits with noteworthy spatiotemporal RNA expression patterns}

Previously, Cuajungco et al. (2007) analyzed expression of 33 TRP channels in an organ of Corti library. In their screen, they were able to detect expression of 19 TRP genes (Table 1). We detected transcripts that encoded 30 TRP subunits in our screen. The difference may have resulted from a more sensitive expression screen in our study or, more likely, because our samples included not only the organ of Corti but also stria vascularis and Reissner's membrane. If the later, we hypothesis that the 11 TRP subunits detected in our screen that were not detected by Cuajungco et al. (2007) may be localized to stria vascularis and Reissner's membrane, i.e., outside the organ of Corti (Table 1).

TRPP channels have gained recent attention as they have several properties that are consistent with the hair cell transduction channel, including large conductance and $\mathrm{Ca}^{2+}$ permeability. Furthermore, TRPP subunits are known to form heteromultimers with different properties and interact with ancillary subunits that could act as linkers between the tip link and the pore-forming subunit (Fettiplace 2009). Cuajungco et al. (2007) detected RNA transcripts for four of five TRPP channels in the organ of Corti. In our screen, we identified several TRPP subunits that had noteworthy expression patterns. TRPP3 (PKD2L1) expression changed dramatically with a spatiotemporal rise of expression that roughly paralleled the developmental acquisition of transduction in the mouse cochlea (Lelli et al. 2009). A developmental basalapical gradient was evident with a delayed rise in expression in the apex and overall higher expression in the apex. TRPP3 also displayed the second largest fold-change in expression of all the TRPs in our screen. As such, we wonder whether TRPP3 may play a role in hair cell transduction. Interestingly, TRPP3 has been shown to interact with alpha actinin which has recently been localized to stereocilia of inner and outer hair cells (Li et al. 2007).

PKD1L3 also displayed a spatiotemporal rise in expression that paralleled the acquisition of transduction and, therefore, may be worthy of further investigation. PKD1L3 and TRPP3 (PKD2L1) have been shown to form heteromeric channels in sensory taste receptor cells and may contribute to sour taste transduction (Ishimaru et al. 2006). It is attractive to speculate that the parallel rise in expression that we note here may indicate an interaction between PKD1L3 and TRPP3 in the cochlea that gives rise to physiologically significant aspects of cochlear function.

Clearly, the data we present here can only call attention to patterns of RNA expression. As such, we feel these data may form the foundation for future follow-up experiments including localization of TRP message and protein, acute, or chronic genetic deletion of TRP sequences followed by evaluation of the consequences for single cell function, auditory organ function, and behavioral assays. Furthermore, since over 30 mouse models have been generated that carry targeted deletions or mutations of single TRP genes, and yet, none have been shown to be necessary for hair cell transduction, we suggest that animals deficient in two or more subunits may yield more fruitful outcomes. Based on our screen, we suggest that animals deficient in TRPA1 and TRPV1 or TRPP3 and PDK1L3 may yield interesting phenotypes.

\section{ACKNOWLEDGEMENTS}

This work was supported by the NIH/NIDCD grants DC008853 (G.S.G.G.); DC05439 (J.R.H.); and the Hazel Thorpe Carmen and George Gay Carmen Trust for Scientific Research (J.R.H.).

\section{REFERENCES}

Abe T, Kakehata S, Kitani R, Maruya S, Navaratnam D, Santos-Sacchi J, SHINKAWA H (2007) Developmental expression of the outer hair cell motor prestin in the mouse. J Membr Biol 215:49-56

Akopian AN, Ruparel NB, Jeske NA, Hargreaves KM (2007) Transient receptor potential TRPA1 channel desensitization in sensory neurons is agonist dependent and regulated by TRPV1-directed internalization. J Physiol 583:175-193

Alessandri-Haber N, Dina OA, Chen X, Levine JD (2009) TRPC1 and TRPC6 channels cooperate with TRPV4 to mediate mechanical hyperalgesia and nociceptor sensitization. J Neurosci 29:6217-6228

Bautista DM, Jordt SE, Nikai T, Tsuruda PR, Read AJ, Poblete J, Yamoah EN, Basbaum AI, Julius D (2006) TRPA1 mediates the inflammatory actions of environmental irritants and proalgesic agents. Cell 124:1269-1282

Belyantseva IA, Adler HJ, Curi R, Frolenkov GI, Kachar B (2000) Expression and localization of prestin and the sugar transporter GLUT-5 during development of electromotility in cochlear outer hair cells. J Neurosci 20:RC116

Caterina MJ, Schumacher MA, Tominaga M, Rosen TA, Levine JD, Julius D (1997) The capsaicin receptor: a heat-activated ion channel in the pain pathway. Nature 389:816-824

Corey DP, Garcia-Anoveros J, Holt JR, Kwan KY, Lin SY, Vollrath MA, Amalfitano A, Cheung El, Derfler BH, Duggan A, Geleoc GS, Gray PA, Hoffman MP, Rehm HL, Tamasauskas D, Zhang DS (2004) TRPA1 is a candidate for the mechanosensitive transduction channel of vertebrate hair cells. Nature 432:723-730

Cujungco MP, Grimm C, Heller S (2007) TRP channels as candidates for hearing and balance abnormalities in vertebrates. Biochim Biophys Acta 1772:1022-1027 
Damann N, Voets T, Nilius B (2008) TRPs in our senses. Curr Biol 18:R880-R889

Di Palma F, Belyantseva iA, Kim HJ, Vogt TF, Kachar B, NobenTrauth K (2002) Mutations in Mcoln3 associated with deafness and pigmentation defects in varitint-waddler (Va) mice. Proc Natl Acad Sci USA 99:14994-14999

Farris HE, LeBlanc CL, Goswami J, Ricci AJ (2004) Probing the pore of the auditory hair cell mechanotransducer channel in turtle. J Physiol 558:769-792

Fettiplace R (2009) Defining features of the hair cell mechanoelectrical transducer channel. Pflugers Arch 458:1115-1123

Fleige S, Pfaffl MW (2006) RNA integrity and the effect on the real time qRT-PCR performance. Mol Aspects Med 27:126-139

Gabashvili IS, Sokolowski BH, Morton CC, Giersch AB (2007) Ion channel gene expression in the inner ear. J Assoc Res Otolaryngol 8:305-328

Grimm C, Cujujungco MP, van Aken AF, Schnee M, Jors S, Kros CJ, RicCI AJ, HeLLER S (2007) A helix-breaking mutation in TRPML3 leads to constitutive activity underlying deafness in the varitintwaddler mouse. Proc Natl Acad Sci USA 104:19583-19588

Grimm C, Jors S, Heller S (2009) Life and death of sensory hair cells expressing constitutively active TRPML3. J Biol Chem 284:13823-13831

Ishimaru Y, Inada H, Kubota M, Zhuang H, Tominaga M, Matsunami H (2006) Transient receptor potential family members PKD1L3 and PKD2L1 form a candidate sour taste receptor. Proc Natl Acad Sci USA 103:12569-12574

Kiselyov K, PATterson RL (2009) The integrative function of TRPC channels. Front Biosci 14:45-58

Kwan KY, Allchorne AJ, Vollrath MA, Christensen AP, Zhang DS, WOOLF CJ, CoRey DP (2006) TRPA1 contributes to cold, mechanical, and chemical nociception but is not essential for hair-cell transduction. Neuron 50:277-289

Lelli A, Asai Y, Forge A, Holt JR, Geleoc GS (2009) Tonotopic gradient in the developmental acquisition of sensory transduction in outer hair cells of the mouse cochlea. J Neurophysiol 101:2961-2973

Li Q, Dai XQ, Shen PY, Wu Y, Long W, Chen CX, Hussain Z, Wang S, CHEN XZ (2007) Direct binding of alpha-actinin enhances TRPP3 channel activity. J Neurochem 103:2391-2400

Liedtke W, Choe Y, Marti-Renom MA, Bell AM, Denis CS, Sali A, Hudspeth AJ, Friedman JM, Heller S (2000) Vanilloid receptorrelated osmotically activated channel (VR-OAC), a candidate vertebrate osmoreceptor. Cell 103:525-535

Montell C (2005) The TRP superfamily of cation channels. Sci STKE 2005:re3
Nagata K, Zheng L, Madathany T, Castiglioni AJ, Bartles JR, Garcia-Anoveros J (2008) The varitint-waddler (Va) deafness mutation in TRPML3 generates constitutive, inward rectifying currents and causes cell degeneration. Proc Natl Acad Sci USA $105: 353-358$

Oberwinkler J, Lis A, Giehl KM, Flockerzi V, Philipp SE (2005) Alternative splicing switches the divalent cation selectivity of TRPM3 channels. J Biol Chem 280:22540-22548

Owsianik G, Talavera K, Voets T, Nilius B (2006) Permeation and selectivity of TRP channels. Annu Rev Physiol 68:685-717

Prober DA, Zimmerman S, Myers BR, McDermott BM Jr, Kim SH, Caron S, Rihel J, Solnica-Krezel L, Julius D, Hudspeth AJ, Schier AF (2008) Zebrafish TRPA1 channels are required for chemosensation but not for thermosensation or mechanosensory hair cell function. J Neurosci 28:10102-10110

Ramsey IS, Delling M, Clapham DE (2006) An introduction to TRP channels. Annu Rev Physiol 68:619-647

Raybould NP, Jagger DJ, Kanjhan R, Greenwood D, Laslo P, Hoya N, Soeller C, Cannell MB, Housley GD (2007) TRPC-like conductance mediates restoration of intracellular $\mathrm{Ca} 2+$ in cochlear outer hair cells in the guinea pig and rat. J Physiol 579:101-113

Salas MM, Hargreaves KM, Akopian AN (2009) TRPAl-mediated responses in trigeminal sensory neurons: interaction between TRPA1 and TRPV1. Eur J NeuroSci 29:1568-1578

Tabuchi K, Suzuki M, Mizuno A, Hara A (2005) Hearing impairment in TRPV4 knockout mice. Neurosci Lett 382:304-308

TAKumida M, ANNiko M (2009) Expression of transient receptor potential channel mucolipin (TRPML) and polycystine (TRPP) in the mouse inner ear. Acta Otolaryngol 27:1-8

Takumida M, Kubo N, Ohtani M, Suzuka Y, Anniko M (2005) Transient receptor potential channels in the inner ear: presence of transient receptor potential channel subfamily 1 and 4 in the guinea pig inner ear. Acta Otolaryngol 125:929-934

van Aken AF, Atiba-Davies M, Marcotti W, Goodyear RJ, Bryant Je, Richardson GP, Noben-Trauth K, Kros CJ (2008) TRPML3 mutations cause impaired mechano-electrical transduction and depolarization by an inward-rectifier cation current in auditory hair cells of varitint-waddler mice. J Physiol 586:4035418

Walker RG, Willingham AT, Zuker CS (2000) A Drosophila mechanosensory transduction channel. Science 287:2229-2234

Wang H, Woolf CJ (2005) Pain TRPs. Neuron 46:9-12

Woolf CJ, Costigan M (1999) Transcriptional and posttranslational plasticity and the generation of inflammatory pain. Proc Natl Acad Sci USA 96:7723-7730 